\title{
Do Binucleate Cardiomyocytes Have A Role in Myocardial Repair? Insights Using Isolated Rodent Myocytes and Cell Culture
}

\author{
Michael J. Stephen ${ }^{1}$, Brian J. Poindexter ${ }^{1}$, Johan A. Moolman ${ }^{2}$, David Sheikh-Hamad ${ }^{3}$ and \\ Roger J. Bick ${ }^{1, *}$ \\ ${ }^{1}$ Department of Pathology, University of Texas Medical School at Houston, Texas, USA, ${ }^{2}$ Department of Medical Physi- \\ ology, Faculty of Health Sciences, University of Stellenbosch, South Africa and ${ }^{3}$ Renal Section, Department of Medicine, \\ Baylor College of Medicine, Houston, Texas, USA
}

\begin{abstract}
Neonatal and adult cardiomyocytes were isolated from rat hearts. Some of the adult myocytes were cultured to allow for cell dedifferentiation, a phenomenon thought to mimic cell changes that occur in stressed myocardium, with myocytes regressing to a fetal pattern of metabolism and stellate neonatal shape.

Using fluorescence deconvolution microscopy, cells were probed with fluorescent markers and scanned for a number of proteins associated with ion control, calcium movements and cardiac function. Image analysis of deconvoluted image stacks and sequential real-time image recordings of calcium transients of cells were made.

All three myocyte groups were predominantly comprised of binucleate cells. Clustering of proteins to a single nucleus was a common observation, suggesting that one nucleus is active in protein synthesis pathways, while the other nucleus assumes a 'dormant' or different role and that cardiomyocytes might be mitotically active even in late development, or specific protein syntheses could be targeted and regulated for reintroduction into the cell cycle.
\end{abstract}

Such possibilities would extend cardiac disease associated stem cell research and therapy options, while producing valuable insights into developmental and death pathways of binucleate cardiomyocytes (word count 183).

\section{INTRODUCTION}

Discussions as to stem cells being the 'global cure' for many diseases continue at a fast pace [1]. However, few positive results with therapeutic promise have been seen, such as in the treatment of Parkinson's disease and cardiac fibrosis (heart attack) [2, 3]. To overcome many ethical concerns in this field of research using fetal stem cells, a great deal of time and effort has been spent recognizing other sources of pluripotential cells, such as cord cells and bone marrow [4]. Further, undifferentiated adult cells have been suggested as promising targets for manipulation and tissue/organ repair $[4,5]$.

It was intriguing therefore when research reports appeared stating that the heart, considered the ultimate postmitotic organ, actually had the potential for regeneration [6, 7]. Based on our observations with binucleate cardiomyocytes, and our hypothesis that one nucleus might be involved in functions such as protein syntheses and ion fluxes, while the other is involved in cell development pathways, we carried out this study.

Both isolated adult cardiomyocytes and cultured neonatal cardiomyocytes were predominantly binucleate, while we

*Address correspondence to this author at the Department of Pathology and Laboratory Medicine, University of Texas Medical School, MSB 2.288, 6431 Fannin Street, Houston, Texas 77030, Tel: (713)-500-5406; Fax: (713)-500-0730; E-mail: Roger.J.Bick@uth.tmc.edu http://pathology.uth.tmc.edu/imaging/index.html have also reported the 'regression' of cultured adult cells to a neonatal form [8]. Therefore, initial experiments attempted to manipulate differentiation of our cultured cardiomyocytes, via additions of various factors, such as angiotensin, and coculturing on different matrices. We hoped that the 'dormant' nucleus would begin functioning in some manner and initiate binucleate myocyte regeneration, our aim being to provide the possibility of an 'instant fix' for fibrotic hearts.

We compared the localization(s) of a number of diverse proteins important in the modulation of intracellular calcium levels in neonatal, adult and cultured (dedifferentiated) adult cardiomyocytes. At the same time we identified markers of cardiomyocyte maturation such as adrenoreceptor numbers and subcellular organelle development [9]. Comparisons of calcium transients in myocyte groups using real-time fluorescent imaging were made, to determine if there was a 'dominant-dormant nuclei pair' with potential induction of the second nucleus into the contraction-relaxation cycle [10]. We began with angiotensin treatments, co-culturing with cardiac fibroblasts and using a complex culture matrix. However, these studies did not produce any additional, revealing observations compared to our normal laminin-based cultures, so our early experimental manipulations are only presented in the discussion.

For many years it has been known that cardiomyocytes exhibit a binucleate morphology [11]. The percentage of ploidy is considerable in isolated neonatal mouse myocytes at what is considered a transitionary stage, a stage able to be influenced by multiple factors such as catecholamines, hy- 
poxia and angiotensin II [12-14]. Our cardiomyocyte cultures have many binucleate cells and a number of theories have developed as to the reason for this second nucleus, such as does it indicate a cardiac pathology, or maybe impending apoptotic cell death? This binucleate form is believed to signal that terminal differentiation has been reached, a "tetraploidy checkpoint", thereby affording protection against cell overgrowth and excess proliferation $[15,16]$. However, our research of nuclear calcium signaling gives this second nucleus a potential further role and possibly an important part in cell growth pathways [17].

\section{METHODS}

\section{Cardiomyocyte Isolations}

Cardiomyocytes were cultured as previously described $[8,18]$. No additions were made to any of the cultures, other than medium, to induce growth.

\section{Neonatal Cardiomyocytes}

Neonatal cardiac myocytes were isolated as previously reported [8, 18], sacrificing 2-3 day-old male rats (Harlan Sprague-Dawley, Harlan Co., IN, USA). The hearts were removed and harvested ventricles were digested with collagenase/pancreatin, separated on a Percoll (Pharmacia, Piscataway, NJ, USA) gradient and cultured on laminin coated cover slips in $35 \mathrm{~mm}$ petri dishes. Culture medium was Dulbecco's Modified Eagles Medium (DMEM; Gibco, Grand Island, NY, USA) containing $5000 \mathrm{U} / 1$ streptomycin and $5000 \mathrm{U} / \mathrm{l}$ penicillin plus $10 \%(\mathrm{v} / \mathrm{v})$ HyClone calf serum (Logan, UT, USA). Myocytes began beating spontaneously after 3-4 days at $37{ }^{\circ} \mathrm{C}$ in 90/10; air/carbon dioxide. Experiments were performed on days 4 and/or 5 .

\section{Isolation and Culturing of Adult Rat Cardiomyocytes}

Adult male rat (Harlan Sprague-Dawley; 200 g) cardiomyocytes were harvested as previously described [8, 18]. Anesthetized rats received 3000 IU of heparin by i.v. injection and the heart was exposed by a longitudinal thoracotomy incision. The thymus and fascia were cleared from the aorta with a sterile swab, the aorta was cross-clamped and cut distally, and the heart was removed and placed in $50 \mathrm{ml}$ of ice-cold Joklik media [1 package of Joklik media powder (Gibco, NY, USA), suspended in 11 water, supplemented with $3.91 \mathrm{~g}$ taurine, $2.0 \mathrm{~g} \mathrm{NaHCO}_{3}, 0.391 \mathrm{~g} \mathrm{I-}$ glutamine and $0.282 \mathrm{~g}$ adenosine]. The aorta was cannulated and flushed with cold Joklik media, perfused at 12$15 \mathrm{ml} / \mathrm{min}$ for 5 minutes, then transferred to a Langendorff apparatus, where it was flushed with warm Joklik media, and digested by perfusion with Joklik media containing $0.1 \%$ collagenase and $0.1 \%$ trypsin for 45 minutes. Ventricles were minced, placed in digestion buffer containing $0.1 \%$ collagenase (in Joklik media, then incubated in a shaking water bath $\left(37^{\circ} \mathrm{C}\right.$ for $\left.30 \mathrm{~min}\right)$ and centrifuged $(3 \mathrm{~min}$, at $50 \mathrm{x}$ g). Pellets were washed twice in $4 \%$ bovine serum albumin (BSA) and once in $2 \%$ BSA solution, then suspended in

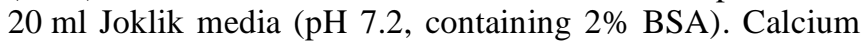
chloride solution was slowly added to a final concentration of $1.25 \mathrm{mM}$. Cells were pelleted at $50 \times \mathrm{x}$ and suspended in $4 \mathrm{ml}$ of warm, serum-free DMEM medium by gentle pipetting. Adult cells begin to contract spontaneously upon addition of medium [8, 18].

\section{Dedifferentiation of Adult Cardiomyocytes}

The cell suspension obtained above (1-2 drops) was layered onto sterile laminin coated cover slips and incubated for 30 minutes $\left(37{ }^{\circ} \mathrm{C}\right.$ in $\left.5 \% \mathrm{CO}_{2} / 95 \% \mathrm{O}_{2}\right)$ to allow cell attachment. Plating media [DMEM containing $10 \%$ fetal bovine serum, 3 (g/ml Ara-C (to inhibit fibroblast growth), 10 (g/ml insulin, and $5 \mathrm{U} / \mathrm{ml}$ each of penicillin and streptomycin] was gently added. Cells were fed fresh plating media every other day and began beating after 5-7 days of incubation. Experiments were carried out between days 7-14. Dedifferentiation is determined by monitoring myocyte hypertrophy and expression of adhesion proteins as previously described [8, 18], as the cells assume a stellate, neonatal-like shape by day 10 . Myofibrillar (re)alignment occurs between days 10-14.

\section{Imaging}

Fluorescently probed cells were scanned with a Fluorescence Deconvolution Microscope (Applied Precision, Issaquah, WA, USA). Image analysis was carried out with SoftWoRxTM software (Applied Precision), with data sets subjected to a minimum of five deconvolution iterations and image stacking. Sequential real-time image recordings of contractile events were made on a Wallac/Perkin Elmer (Gaithersburg, MD, USA) Concord fluorescence spectrophotometer (Melville, NY, USA; 3) to show a number of contraction-relaxation cycles (minimum of 3-5), and video recordings of these events were made [18]. Fluorescence (pixel densities) was measured using both Corel Paint 10 (Ottawak, Ontario, Canada) and Sigma Scan Pro 5.0 (SPSS, Chicago, IL, USA) software.

All animal usage procedures were approved by the UTHSC Animal Welfare Committee (Protocols HSC-AWC05-143 and 144), following NIH guidelines.

\section{RESULTS}

Fig. (1) shows images of binucleate cardiomyocytes from each of the three isolates studied; neonatal stage (top), adult (middle) and cultured-dedifferentiated adult (bottom). Images were acquired by deconvolution fluorescence microscopy at a magnification of $x 600$. Note that when adult cells are cultured, they increase in size (hypertrophy), demonstrate fiber randomization and assume a state between neonatal (stellate) and adult (rods) cardiomyocytes. The blue stain identifies nuclei (DAPI; two nuclei in each of the cells), the green is cardiac actin (tagged Phallocidin probe). The red stain identifies adrenoreceptors (Texas Red) that are visualized to track maturation of the cells, as previously mentioned in the Methods section.

To recognize the localizations of various proteins as myocyte development occurs, Fig. (2) shows the IP3 receptors in a dedifferentiated cardiomyocyte, with the yellow protein tightly clustered around one of the two nuclei. However, in the right panel, the adult cardiomyocyte shows the $\mathrm{Na}^{+}+\mathrm{K}^{+}$ATPase pump protein (yellow) distributed across the cell surface with no clustering to a particular nucleus. Once again, binucleate cells are shown, proteins are yellow, nuclei are blue and cardiac actin is green in the left panel and red in the right panel, a necessary change due to the wavelength of the fluorescent tags being used. 


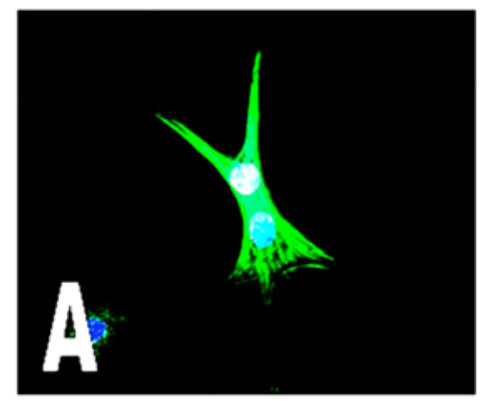

Neonate

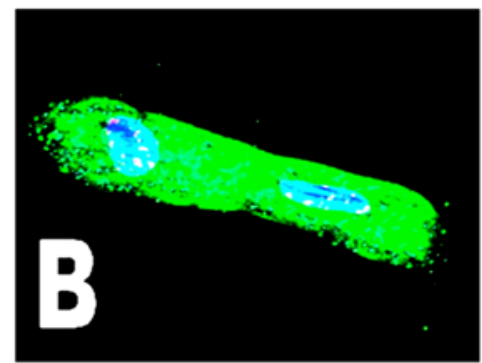

Adult

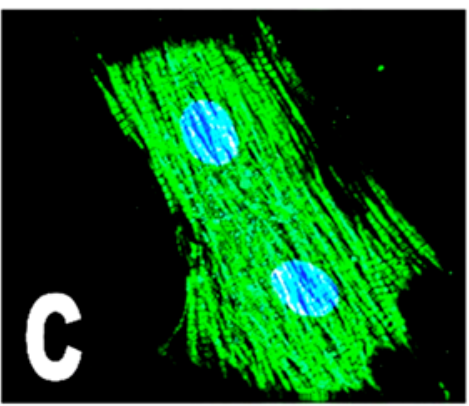

\section{Dedifferentiated}

Fig. (1). Images of binucleate cardiomyocytes from the three isolates studied, acquired by deconvolution fluorescence microscopy as described (magnification of x600). The blue stain identifies nuclei (DAPI; two nuclei in each of the cells), the green is cardiac actin (Phallocidin) and the red are the protein, in these particular images, the alpha adrenoceptor. $\mathrm{A}=$ Neonate; $\mathrm{B}=\mathrm{Adult} ; \mathrm{C}=\mathrm{Cultured}$ (dedifferentiating) Adult (Mag. X 600).

Fig. (3) shows a number of probes for various proteins in binucleate cells and again demonstrates protein clustering about one nucleus. Panel A shows a freshly isolated adult cardiomyocyte in which one (upper) blue nucleus sits on its own with no associated calcitonin gene related protein (CGRP-red). Panel B is a dedifferentiating myocyte with NFKB65 clustered around the upper nucleus, and even demonstrates the contractile protein actin being predominantly associated with the same nucleus. Panel C shows NFkB50 (NF-kappa B50) localized to the upper nucleus and Panel D is a dedifferentiating cell with a probe for alpha-adrenoreceptors shown clustered only around the upper nucleus, possibly demonstrating rearrangement of the cellular components and contractile proteins during differentiation, or changes in protein syntheses.

We considered that if binucleation was as common in cardiomyocytes in the heart, as was seen in the cultured cells, then the reported myocyte regression in cardiac dysfunction, coupled with our observations here, might direct our attention to the presence of complementary pathways in cardiac repair and recovery. Fig. (4) is a model of multiple, stacked sections from a core sample of human myocardium before ventricular unloading with an assist device [19, 20]. Note the clustering of adrenoceptor protein around only one nucleus (single white arrow), and not the other (double arrow).

To further investigate the role(s) of the two nuclei, we employed real time fluorescent spectrophotometric imaging to see if calcium transients in neonatal and dedifferentiated cells emanated from one, or both, nuclei. Fig. (5) is a sequence of images from a cultured adult dedifferentiated cell. Note the two nuclei in frame 1 indicated by the arrows. In frame 3 the arrows point to 'hot spots' or 'sparks', with one of the two nuclei beginning to load with calcium. However, in frames 4 through 6 , the other nucleus is the one that actually 'fires' to initiate contraction. Frames 7 through 9 are at the beginning of another cycle and once again show sparks (frame 8) and one nucleus firing (frame 9). This singlenucleus activity is also seen in binucleate neonatal cardiomyocytes.

However, as shown in Fig. (6), calcium transient initiation in freshly isolated adult cells is from one end, with the
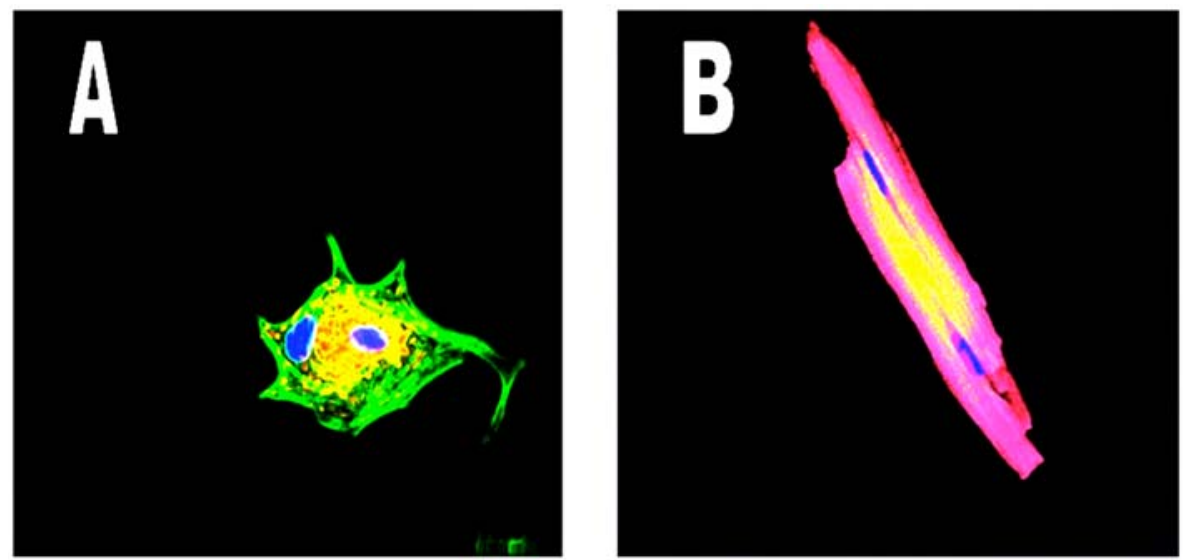

Fig. (2). Shows the IP31 receptors in a dedifferentiated cardiomyocyte (left panel, yellow) tightly clustered around one of the two nuclei while in the adult cardiomyocyte (right panel) however, the yellow $\mathrm{Na}^{+}+\mathrm{K}^{+}$ATPase pump protein does not demonstrate clustering Nuclei are blue, proteins are yellow and cardiac actin is green in the left panel and red in the right panel, due to different wavelength tags being used. (Mag. Ax900; Bx300). 

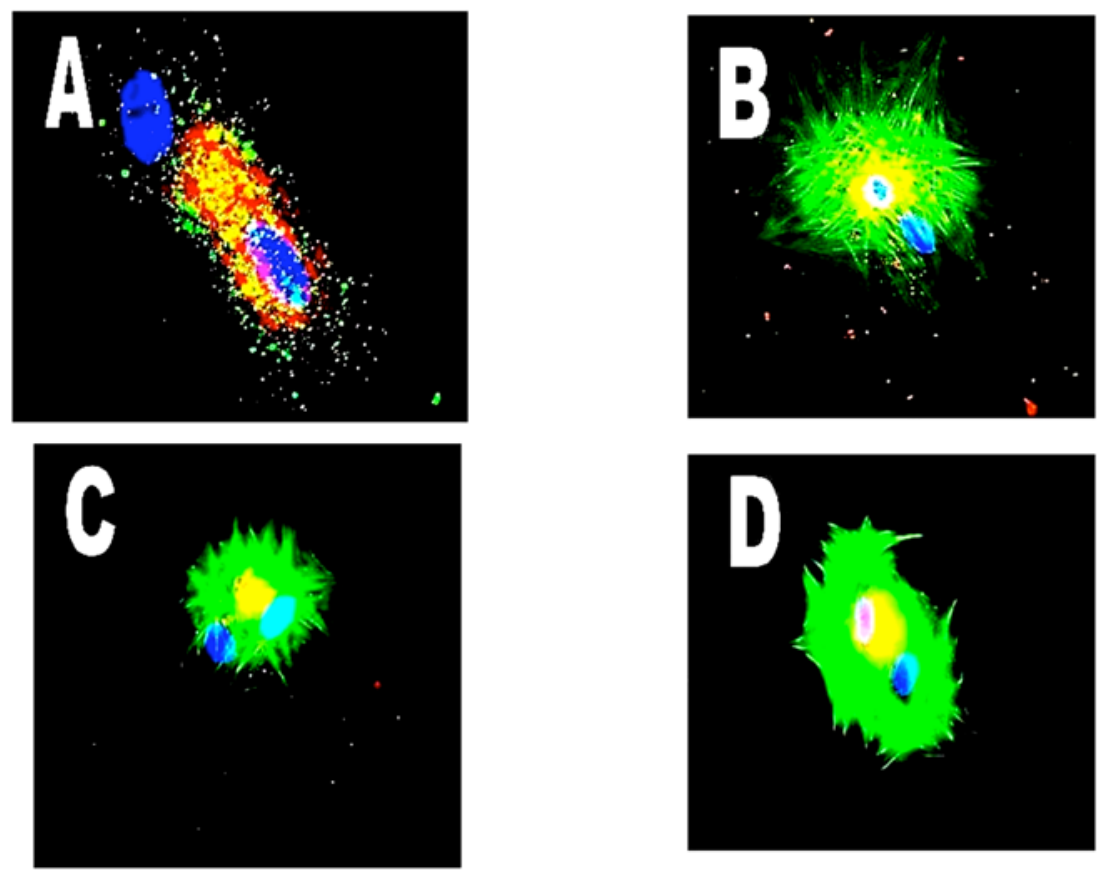

Fig. (3). Shows clustering of a number of probes for various proteins in binucleation. Panel A - freshly isolated adult cardiomyocytes and CGRP (red). Panel B - dedifferentiating myocyte with NFkB65. Panel C is NFkB50 in a dedifferentiating cell probed for alpha-adrenoreceptors. (Mag. X 600).

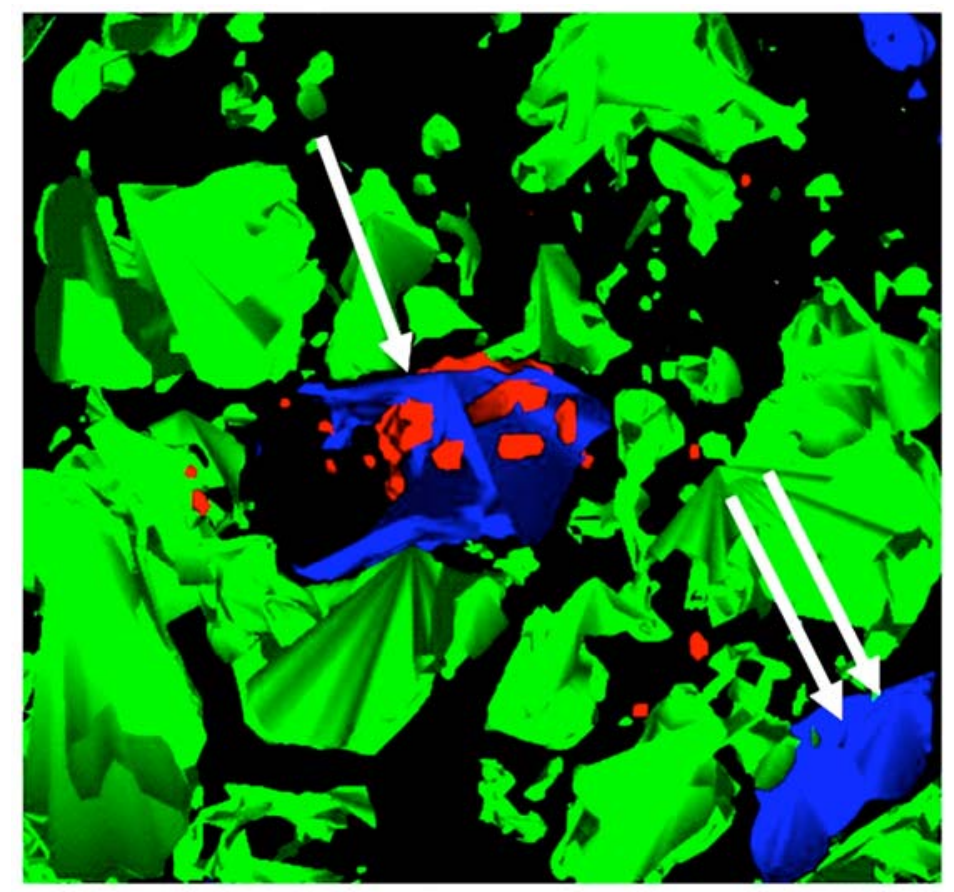

Fig. (4). Model generated from multiple, stacked sections of fluorescence deconvolution acquisitions of a core sample of human tissue showing clustering of alpha-adrenoceptor protein around only one nucleus (single white arrow), and not with the other intramyocytic nucleus (double arrow).

wave traveling the length of the myocyte before dissipating (frame 3) and restarting (frame 4). There is no involvement of the nuclei other than an accumulation of calcium as the wave passes through the cell. Further videos are available at http://www.uth.tmc.edu/pathology/research/corelab/vids.htm 1.

\section{DISCUSSION}

Manipulation of in-situ adult cells would be a minimally invasive therapy, overcoming rejection associated problems. An understanding of differentiation in our cultured cardiomyocytes using diverse manipulations such as cytokine treatments, co-culturing with fibroblasts, using different cul- 
1

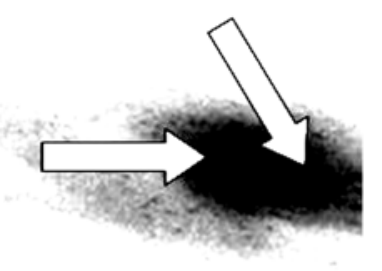

2

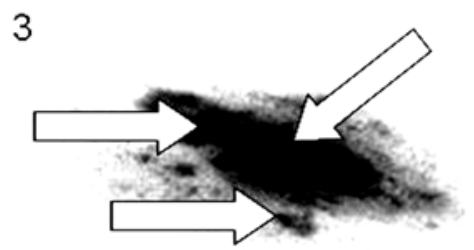

4

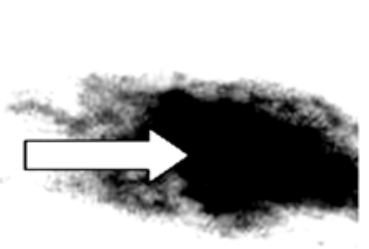

5

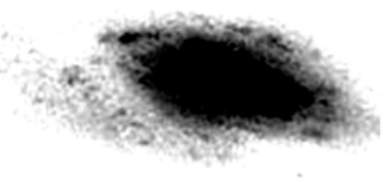

6

7
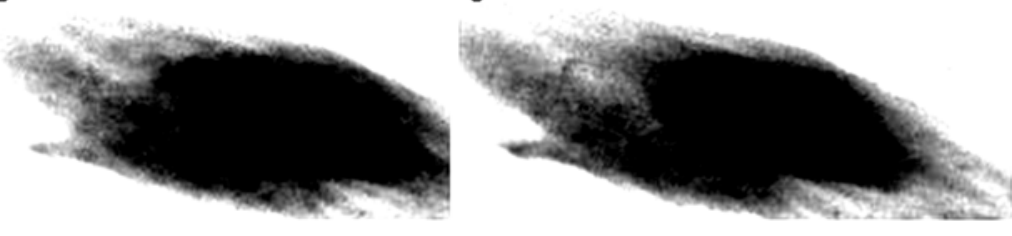

8

9
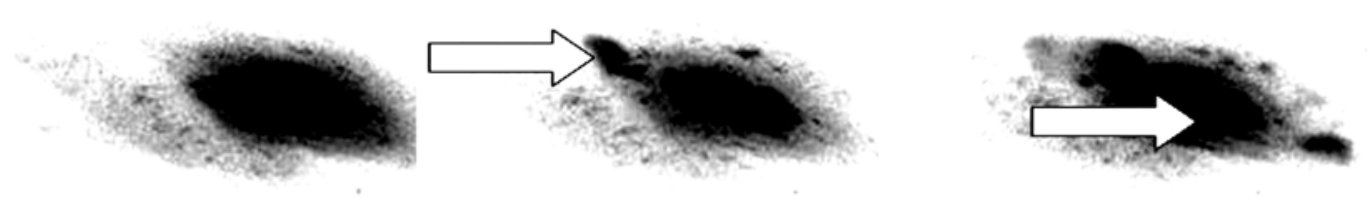

Fig. (5). Sequence of images from a cultured adult dedifferentiated cell using FLUO 4 as the calcium sensitive probe. Note the two nuclei indicated by the arrows in frame 1, the arrows show 'hot spots' or 'sparks' in frame 3. In frames 4 through 6 , 'firing' of one of the nuclei can be seen. Frames 7 through 9 demonstrate the beginning of another cycle, with sparking in frame 8 and one nucleus firing in frame 9 and this is seen in binucleate neonatal cardiomyocytes as well.

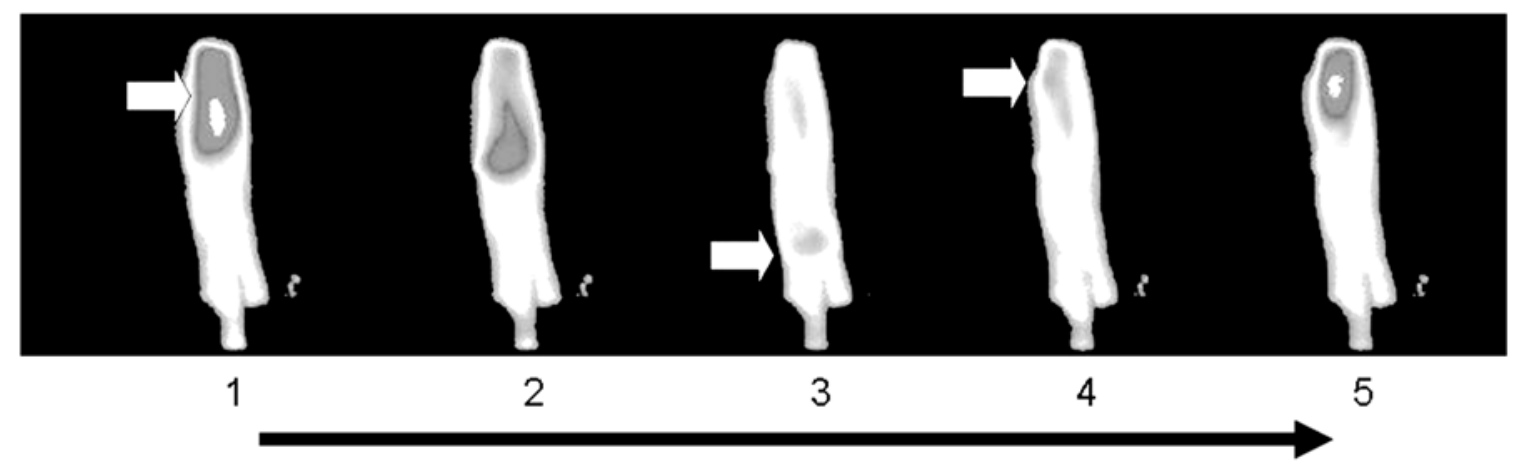

Fig. (6). Shows a sequence of images of freshly isolated adult cardiomyocyte, captured by real-time fluorescence spectrophotometry. The probe is FLUO 4. Highest concentrations of calcium are seen as bright white areas. Captures were made every 43 milliseconds.

ture matrices (simple $\mathrm{v}$ complex), exposure to angiotensin, etc, was hoped for and that the hypertrophic responses could be somehow controlled with a potential for utilization in tissue replacement. This was appealing and cardiomyocyte binucleation suggested the possibility that adult cardiomyocytes may not be at a developmental end-point [21, 22] and would allow us some differentiation/maturation control. However, after finding that angiotensin induced fibroblasts secrete factors that stimulated myocyte growth $(16.7 \pm 1 \%$ increase in angiotensin treated neonatal cardiomyocyte protein content, $\mathrm{n}=18, \mathrm{p}<0.05$ ), we subsequently found that these factors did not in fact condition myocytes to further responses to increased exogenous angiotensin [23]. Furthermore, a fibroblast-derived matrix (cardiogel) did support rapid maturation of cultured myocytes [24, 25], but changes in calcium signaling mechanisms in response to angiotensin treatments were entirely too inconsistent. Therefore, responses to angiotensin treatments was not a line of investigation that gave us much hope in proving our hypothesis that using cultured cells as an infusible repair of a cardiac infarct and subsequent fibrosis was a possibility, and it was not pursued.

Our interest was again piqued regarding manipulation of myocyte growth following our observations that most of our isolated myocytes were binucleate at all three stages of development (neonatal, adult, and differentiated; >95\%). We further discovered that only one nucleus was the focus of calcium transients in most neonatal and dedifferentiated cardiomyocytes and this observation, coupled with the observation that many of the proteins we routinely visualized were predominantly associated with only one nucleus, led us to 
hypothesize that during maturation (neonates) and reorganization (dedifferentiated) these specific protein localizations and particular protein syntheses, were integral parts of cellular maturation and development, involving only one of the two nuclei. We weren't too surprised by these results in neonates and dedifferentiating cells, but similar findings in mature, adult, rod-shaped cardiomyocytes directed our attention to the possibility of there being specific roles for each nucleus in all stages of cardiomyocyte maturation.

Biochemical pathways associated with these findings are not reported here and further work in this area is underway. However, these are important and novel observations that lend support to earlier work reporting cardiomyocyte proliferation in pathologic conditions, and mature cardiomyocytes having the potential for further mitosis [6]. One line of thought is that one nucleus is involved in myocyte maturation (size), while the other is involved in specific mechanisms that increase myocyte numbers as needed, even though increased cell numbers do not always result. This could explain the homogenous distribution of some (sarcolemmal) proteins such as the $\mathrm{Na}^{+}+\mathrm{K}^{+}-$ATPase (sodium potassium adenosine triphosphatase; EC 3.6.3.9), while the intracellular calcium channel regulators stanniocalcin, and the $\mathrm{IP}_{2}$ and $\mathrm{IP}_{3}$ gated proteins, remain associated with a single nucleus to modulate nuclear calcium fluxes [8].

This research has provided results supporting previous studies which indicate that binucleation occurs at a time when ionic changes are necessary to take the myocytes from a neonatal to adult stage and when adult proteins and hypertrophic pathways are needed and initiated. As this development process requires the calmodulin dependent kinase and IP3 receptors, it is localized to the nucleus and involves luminal calcium control via interconnections of the sarcoplasmic reticulum and nuclear envelope. These findings are therefore consistent with, and add evidence to, membranous excitation-transcription coupling as has previously reported [25-27].

We have demonstrated specificity of protein localization and protein synthesis to a single nucleus in isolated binucleate cardiomyocytes; we have also revealed that proteins required to interact with the extracellular milieu, such as the $\mathrm{Na}^{+}+\mathrm{K}^{+}$-ATPase, do not exhibit this focused distribution. These observations pose the question of whether the 'dormant' second nucleus could be 'switched on' by appropriate compounds and become involved in cell replication and myocardial regeneration. This question is especially relevant, as cardiomyocyte dedifferentiation and fetal phenotype expression does not reactivate the second nucleus. However, it has been shown, in dividing rat cardiomyocytes that exposure to catecholamines can drive the cells to proliferate rather than enlarge and that adult rat cardiomyocytes do contain the proliferating cell nuclear antigen [28].

Our findings support those of $\mathrm{Wu}$ and colleagues [29], concluding (i) nuclear calcium is required for protein formation, (ii) cytoplasmic calcium is necessary for cell contraction and (iii) specific ionic requirements occur as the myocytes mature and/or differentiate. We suggest that one nucleus is predominantly involved in contractile function in non-mature, binucleate cardiomyocytes, while protein syntheses are also localized to a single (other) nucleus, and these specific roles are seen via one nucleus having continued increased intra-nuclear calcium, while the other had fluctuating levels. A number of possibilities for cell proliferation initiation come to mind, such as death of the 'active' nucleus inducing the 'dormant', second nucleus to become active, or perhaps the 'second' nucleus is involved in apoptosis and not necrosis? Are the two nuclei signaling to each other? All these considerations require extensive investigation in case adult cardiomyocyte proliferation can and does occur and can be controlled.

Here we again mention that cultured-dedifferentiating adult cells revert to a neonatal-like dependence on nuclear calcium for contraction, and do return to fetal protein synthesis, adaptations that have been suggested to occur in heart failure [30]. Perhaps this reversion is a re-initiation of processes to stop mitosis, thereby encouraging older cardiomyocytes to continue replicating, thereby offering us a new treatment target. Whether cardiomyocytes have the potential to regenerate is a matter for intense research, while methods to goad the heart into a regenerative state, with without stem cell treatments, is of immense importance [31]. Binucleate cells function independently, and carry out diverse roles and signaling associated with a young stage of development [32], but human hearts do return to a fetal-like form in disease and stress, perhaps in a (futile?) effort to restart cell division, the regeneration process [30] and adaptation [7].

Stem cells show promise in the treatment of cardiac disease, but our own tissue might have more of a say in cardiac repair than we at first believed. The heart certainly often recovers with ventricular unloading and perhaps it is not too far-fetched to envision ventricular unloading being coupled with targeted protein syntheses as a way to directing cardiomyocytes to reacquire a fetal phenotype and to channel this change into a replenishment of functional, contracting, muscle cells, overcoming areas of fibrosis, or as an adjunct to pharmacologic and mechanical unloading [33-38].

\section{REFERENCES}

[1] Dobson R. The cure for all our ills? Stem-cell therapy offers the prospect of revolutionary treatments for everything from broken bones and diabetes to cancer. Belfast Telegraph 2006 Aug 1. Available from http://www.encyclopedia.com/doc/1P2-10733960. html,

[2] Langston JW. The promise of stem cells in Parkinson disease. J Clin Invest 2005; 115: 23-5.

[3] Dennison JA. Stem-Cell study may point to cure for heart attacks. 2005 July 26. Available from http://health.dailynewscentral.com/ content/view/0001354/44/.

[4] $\mathrm{Xu} \mathrm{YQ,} \mathrm{Liu} \mathrm{ZC.} \mathrm{Therapeutic} \mathrm{potential} \mathrm{of} \mathrm{adult} \mathrm{bone} \mathrm{marrow} \mathrm{stem}$ cells in liver disease and delivery approaches. Stem Cell Rev 2008; 4: 101-12.

[5] Mothe AJ, Kulbatski I, Parr A, Mohareb M, Tator, CH. Adult spinal cord stem/progenitor cells transplanted as neuroshperes preferentially differentiate into oligodendrocytes in the adult rat spinal cord. Cell Transplant 2008; 17: 735-51.

[6] Beltrami AP, Urbanek K, Kajustra J, et al. Evidence that human cardiac myocytes divide after myocardial infarction. New Engl J Med 2001; 344: 1750-57

[7] Lerch R, Tardy-Cantalupi I, Papgeorgiou I, Montessuit C. Cellular recovery after ischemia: physiopathologic aspects. Arch Mal Coeur Vaiss 1997; 90: 17-21.

[8] Poindexter BJ, Smith JR, Buja LM, Bick RJ. Calcium signaling mechanisms in dedifferentiated cardiac myocytes: comparison with neonatal and adult cardiomyocytes. Cell Calcium 2001; 30: 373-82.

[9] Evans HJ, Sweet JK, Price RL, Yost M, Goodwin RL. Novel 3D culture system for study of cardiac myocyte development. Am J Physiol Heart Circ Physiol 2003; 285: H570-8. 
[10] Inui M, Kimura Y, Sasaki T, Tada M. Molecular mechanism of calcium uptake and release by cardiac sarcoplasmic reticulum. Jpn Circ J 1990; 54: 1185-91.

[11] Lourd AV, Anversa P, Giacomelli F, Wiener J. Absolute morphometric study of myocardial hypertrophy in experimental hypertension. I. Determination of myocyte size. Lab Invest 1978; 38 : 586-96.

[12] Shannon R, Chaudhry M. Effect of alpha1-adrenergic receptors in pathophysiology. Am Heart J 2006; 152: 842-50.

[13] Julian RJ. The response of the heart and pulmonary arteries to hypoxia, pressure and volume. A short review. Poult Sci 2007; 86: 1006-11.

[14] Domenighetti AA, Wang Q, Egger M, Richards SM, Pedrazzini T, Delbridge LM. Angiotensin II-mediated phenotypic cardiomyocyte remodeling leads to age-dependent cardiac dysfunction and failure. Hypertension 2005; 46: 426-32.

[15] Sundgren NC, Giraud GD, Stork JPS, Maylie JG, Thornburg KL. Angiotensin II stimulates hyperplasia but not hypertrophy in immature ovine cardiomyocytes. J Physiol 2003; 548: 881-91.

[16] Jonker SS, Zhang L, Louey S, Giraud GD, Thornburg KL, Faber JJ. Myocyte enlargement, differentiation and proliferation kinetics in the fetal sheep heart. J Appl Physiol 2007; 102: 1130-42.

[17] Moavec M, Turek Z, Moravec J. Persistence of neoangiogenesis and cardiomyocyte divisions in right ventricular myocardium or rats born and raised in hypoxic conditions. Basic Res Cardiol 2002; 97: 153-60.

[18] Poindexter BJ, Feng W, Dasgupta A, Bick RJ. Oleandrin produces changes in intracellular calcium levels in isolated cardiomyocytes; a real-time fluorescence imaging study comparing adult to neonatal cardiomyocytes. J Toxicol Environ Health 2006 70: 568-74.

[19] Schnee PM, Shah N, Bergheim M, et al. Reversal of myocyte hypertrophy by ventricular unloading; Cardiac improvement without adrenergic receptor up-regulation and relocalization. MedGenMed 2006; 8: 45 .

[20] Bick RJ, Poindexter BJ, Buja LM, et al. Alterations in cardiomyocytes following left ventricular unloading. Rec Res Dev Membr Biol 2002; 1: 1-10.

[21] Bearzi C, Rota M, Hosoda T, et al. Human Cardiac stem cells. Proc Natl Acad Sci USA 2007; 104: 14068-73

[22] Beltrami AP, Barlucchi L, Torella D, et al. Adult cardiac stem cells are multipotent and support myocardial regeneration. Cell 2003; 114: 763-76

[23] Sen PSS. Angiotensin II and myocyte growth. Role of fibroblasts. Hypertension 1997; 30: 209-16.
[24] Bick RJ, Snuggs MB, Poindexter BJ, Buja LM, Van Winkle WB. Physical, contractile and calcium handling properties of neonatal cardiac myocytes cultured on different matrices. Cell Adhes Commun 1998; 6: 301-10.

[25] Davis RA, Van Winkle WB, Buja LM, Poindexter BJ, Bick RJ. The effect of a simple versus a complex matrix on the polarity of cardiomyocytes in culture. J Burns Wounds 2006; 5: 16-25.

[26] Wu X, Zhang T, Bossuyt J, et al. Local InsP ${ }_{3}$-dependent perinuclear $\mathrm{Ca}^{2+}$ signaling in cardiac myocyte excitation-transcription coupling. J Clin Invest 2006; 116: 675-82.

[27] Nadal-Ginard B, Anversa P, Kajstura J, Leri A. Cardiac stem cells and myocardial regeneration. Novartis Found Symp 2005; 265: $155-7$

[28] Guatimosim S, Amaya MJ, Guerra MT, et al. Nuclear Ca2+ regulates cardiomyocyte function. Cell Calcium 2008; 44: 230-42.

[29] Zhao H, Pestove NB, Korneenko TV, Shakhparonov MI, Modyanov NN. Accumulation of beta (m), a structural member of the $\mathrm{X}, \mathrm{K}$-ASTPase beta-subunit family, in nuclear envelopes of perinatal myocytes. Am J Physiol Cell Physiol 2004; 286: C757-67.

[30] Luo D, Yang D, Lan X, et al. Nuclear Ca2+ sparks and waves mediated by inositol 1,4,5-triphosphate receptors in neonatal rat cardiomyocytes. Cell Calcium 2008; 43: 165-74.

[31] Zhang H, Lou D, Shan Z. Correlation of expression of connexin 43 and cardiomyocyte hypertrophy. Chin J Pathol 1998; 27: 436-8.

[32] Rajabi M, Kassiotis C, Razeghi P, Taegtmeyere H. Return to fetal gene program protects the stressed heart: a strong hypothesis. Heart Fail Rev 2007; 12: 331-43.

[33] Chen X, Wilson RM, Kubo H, et al. Adolescent feline heart contains a population of small, proliferative ventricular myocytes with immature physiological properties. Circ Res 2007; 100: 536-44.

[34] Birks EJ, Tansley PD, Hardy J, et al. Left ventricular assist device and drug therapy for the reversal of heart failure. $\mathrm{N}$ Engl $\mathrm{J}$ Med 2006; 355: 1873-84

[35] Rivello HG, Meckert PC, Vigliano C, Favaloro R, Laguens RP. Cardiac myocyte nuclear size and ploidy decrease after mechanical support. Cardiovasc Pathol 2001; 10: 53-7.

[36] Klotz S, Naka Y, Oz MC, Burkhoff D. Biventricular assist deviceinduced right ventricular reverse structural and functional remodeling. J Heart Lung Transplant 2005; 24: 1195-201.

[37] Frazier OH, Myers TJ, Gregoric I. Biventricular assistance with the Jarvik FlowMaker: A case report. J Thorac Cardiovasc Surg 2004; 128: 625-6.

[38] Leewenburgh BP, Helbing WA, Wenink AC, et al. Chronic right ventricular pressure overload results in hyperplastic rather than a hypertrophic myocardial response. J Anat 2008; 212: 286-94. 